\title{
Debate
}

\section{Scar revision is a useless operation (Opposing the statement)}

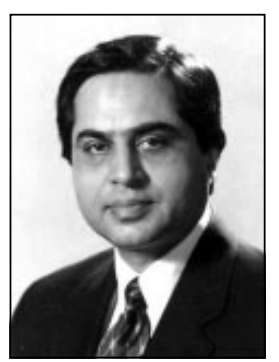

\section{Rajeev B. Ahuja}

Head, Department of Burns and Plastic Surgery

Address for correspondence: Dr. Rajeev B. Ahuja, Head, Department of Burns \& Plastic Surgery, Lok Nayak Hospital and associated Maulana Azad Medical College, India. E-mail: rbahuja@vsnl.com

\section{INTRODUCTION}

ndividuals bearing scars deemed functionally or cosmetically undesirable often seek the services of a plastic surgeon to eliminate the scars and to restore the tissue to its pre injury state. If scar revision is being visualised as a scar elimination procedure, the results are bound to be disappointing, rendering the surgery 'useless'. Mercifully, all plastic surgeons learn in the first couple of months of their training that the goal of scar revision is to improve upon appearance and function, not eliminate the scar! Then, the foremost task of the surgeon is to inform the patient that elimination of scars is almost impossible, but that improvement is realistic. A patient with realistic expectations is more likely to be a satisfied patient. Thus, scar revision is a wellestablished procedure. Having stated this, achievement of satisfying long-term results from scar revision may still present a challenge.

The focus, therefore, shifts to selection of a procedure(s) to render the scar least conspicuous. It is of paramount importance that the type of scar be properly 'classified' on initial examination so that the most appropriate method(s) of treatment can be chosen. Scars present with a spectrum in relation to their number, size, shape, location, orientation, quality, texture, age at presentation, and their relation to each other. Whereas it is fairly easy to learn the surgical principles and the procedures with respect to scar revision the underlying 'Art' is difficult to teach. In a nutshell, like for all 'Art forms', it requires some talent and honing of skills. In the fraternity of surgical tailors a plastic surgeon is like a darner. He repairs an expensive piece of clothing with masterly skills so that the repaired portion is inconspicuous. Even darners can be graded one to ten!

While there can be no 'cook book' approach to scar revision, the general principles require diligent caring. If the patient was under care for primary injuries the skill with which the injuries are repaired goes a long way in minimising the need for subsequent scar revision. For facial lacerations it would imply fitting the 'jig saw' by placing key sutures after most essential and sparing debridement/freshening of margins. Suture tension should have 'room' for postoperative oedema. Tissue loss should be compensated by split-skin grafting and not by extensive mobilisation of local tissues. Also, no attempt is made at reorienting the suture line. Subsequently, non-surgical scar modulation techniques of using silicon gel sheet and pressure (wherever applicable) along with judicious use of 'intralesional' steroids are essential prophylactic measures.

Ideally, scars are revised after they have matured and 
become supple. Scars are not revised in children until growth has completed, or they are severely deforming or limiting function. It cannot be over emphasised that any revision modality should cause no further harm. Obviously, techniques, which recruit local normal tissue to rectify scars, will give the best results and substituting a scar with a full or partial thickness skin graft, or distant flaps, does not fall in the same category. These modalities are reserved for extensive scarring following burns or a major skin loss after trauma. In terms of 'value' they can result in significant improvement of appearance and function- a far cry from being useless!

For 'other' scars, revision modalities include surgical (scalpel) manoeuvring or skin resurfacing with lasers or dermabrasion. Resurfacing offers a camouflage and lasers have added a fresh dimension to this modality. While it is beyond the scope of this deliberation to delve into the virtues or failings of different lasers, it can be summarised that a pulsed dye laser (PDL) is the most appropriate system for treating hypertrophic scars, keloids, erythematous scars, and striae. The PDL carries a low risk of side effects and complications when operated at appropriate treatment parameters and time intervals. Atrophic scars and acne scars are best treated with ablative $\mathrm{CO} 2$ and Er: YAG (erbium: yttriumaluminum-garnet) lasers; however, proliferative keloids and hypertrophic scars should not be vaporised

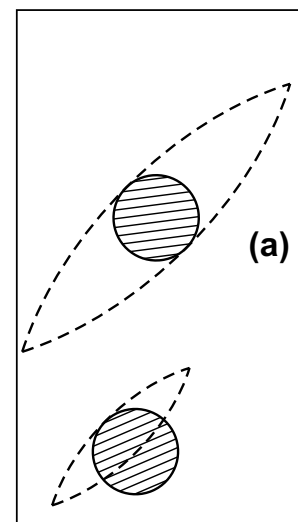

(b)

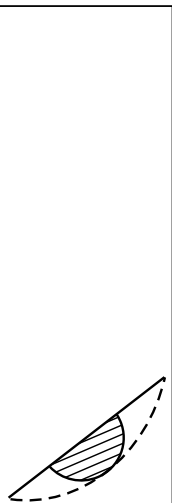

(c)
Figure 1: Large elliptical excisions can be avoided by excising the scar serially, as shown. because of the high risk of scar recurrence or progression. The appropriate choice and use of lasers can significantly improve most scars. Dermabrasion offers the advantage of being a tried-and-true technique familiar to surgeons but in coming years it runs the risk of losing to lasers.

Face offers the best target area to discuss scar revisions carried out surgically. Each millimetre size, location, number and shape of the lesion counts on face because there are many anatomical landmarks. Surgically, scars can be revised by excision (in a single stage or serially) and closed primarily. Needless to say, this would offer the best revision possibility if the ultimate scar can be placed in a crease line and the final scar length is not $>30-40 \%$ of the original scar. Alas, large elliptical excisions often render the scar revision useless! In this regard, Figure 1 and Figure 2 illustrate the value of decision-making. ' $W$ ' excisions and large ' $Z$ '-plastys are not to be used cavalierly in scar revisions. They not only increase the final scar length but also leave indelible mark of 'surgical stamping'. Some examples to illustrate the application of ' $W$ '-plasty, ' $Z$ '-plasty and a lazy ' $\mathrm{S}$ ' design in scar revisions is shown in Figure 3 and Figure 4. Of all other local flaps, the Limberg flap is the most abused flap in scar revision. It makes the scars 'kite shaped' and often leads to pin cushioning on the face. Still, areas like alar base, medical canthus and temples present ideal locations for such a flap.

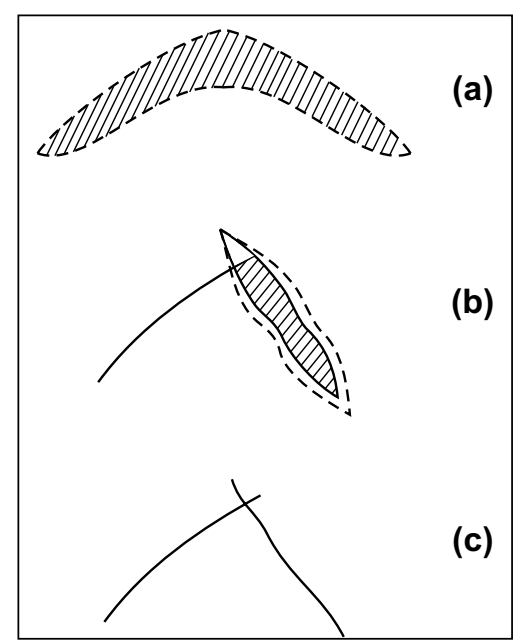

Figure 2: Excising a ' $V$ ' shaped scar in a single stage can often lead to scar hypertrophy because of tension on the ' $\mathrm{V}$ ' flap in two directions and the additional effect of gravity. Excising the two limbs of the scar serially can sometimes help in circumventing this problem. 


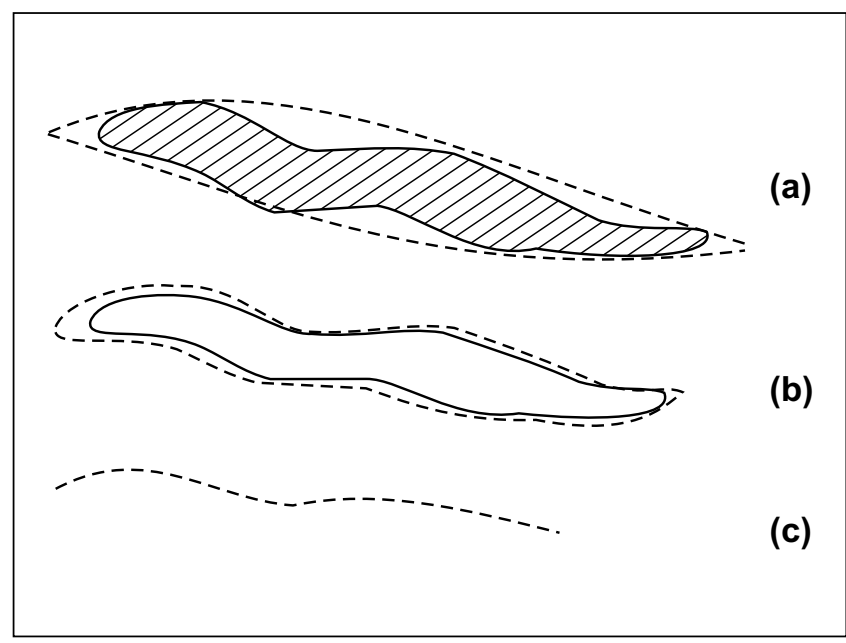

Figure 3: An irregular but linearly oriented scar may be excised elliptically if the final scar lies in a crease line. In any other orientation it may be excised along the scar outline and closed as a wavy line.

Circular scars (lesions) often present other varied challenges than discussed above. Five to six small circular moles/scars (2-3mm size) are best excised and allowed to contract than excising and closing elliptically. Contraction always takes place perpendicular to the line of minimum extensibility and renders the final scar inconspicuous.

Suturing techniques, suture material and needle size, also have a bearing on the final result. This is where quality of darning counts. Precise subdermal approximation with a 'long term' absorbable material prevents stretch due to tension and facilitates skin suturing. Whenever possible,

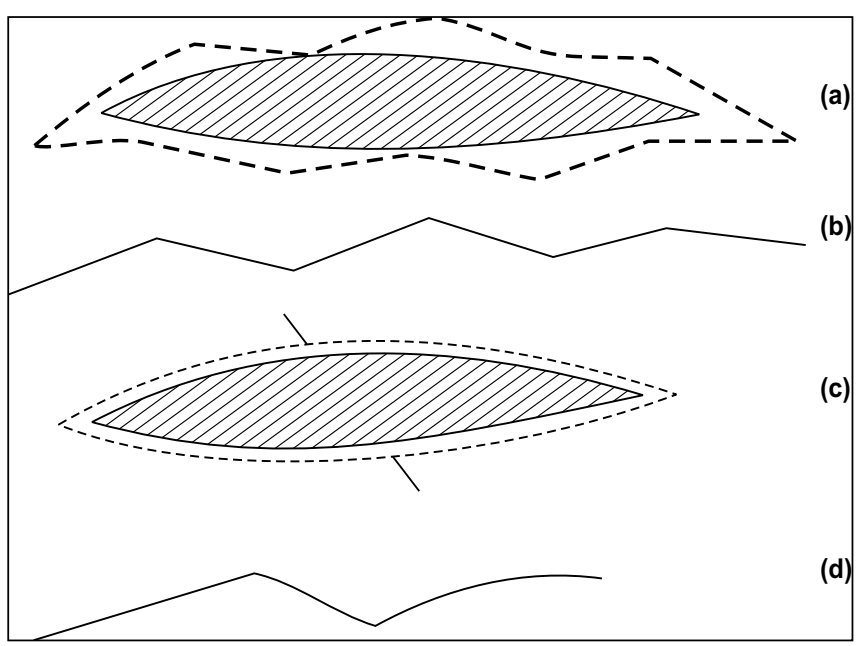

Figure 4: If following an elliptical excision the linear scar is likely to contract then a small ' $Z$ '-plasty can be interposed to break the linear scar. This lengthens the scar much less than a 'W'-plasty.

subcuticular suturing with a pull out $4 / 5$ 'O' nylon/ polypropelene offers best results in scar revisions. 'Glueing' can also curtail surface suturing but requires precise dermal approximation. Dispassionate and unrestrained 'poking' will result in 'pokemons' and separate boys from patient men! No amount of 'creaming' can help in diminishing the blemish but sometimes resurfacing a 'finally' revised scar helps. Realistic time periods should be projected and prophylactic measures, wherever applicable, are helpful utilities.

I hope all the proponents can now revise the scars better! 